\title{
A mechanical property evaluation of graded density Al-Si10-Mg lattice structures manufactured by selective laser melting
}

\author{
I. Maskery ${ }^{\mathrm{a}, *}$, N.T. Aboulkhair ${ }^{\mathrm{a}}$, A.O. Aremu ${ }^{\mathrm{a}}$, C.J. Tuck ${ }^{\mathrm{a}}$, I.A. Ashcroft ${ }^{\mathrm{a}}$, \\ R.D. Wildman ${ }^{a}$, R.J.M. Hague ${ }^{a}$ \\ ${ }^{a}$ Additive Manufacturing 83 3D Printing Research Group, Faculty of Engineering, \\ University of Nottingham, Nottingham NG7 2RD, UK
}

\begin{abstract}
Metal components with applications across a range of industrial sectors can be manufactured by selective laser melting (SLM). A particular strength of SLM is its ability to manufacture components incorporating periodic lattice structures not realisable by conventional manufacturing processes. This enables the production of advanced, functionally graded, components. However, for these designs to be successful, the relationships between lattice geometry and performance must be established. We do so here by examining the mechanical behaviour of uniform and graded density SLM Al-Si10-Mg lattices under quasistatic loading. As-built lattices underwent brittle collapse and non-ideal deformation behaviour. The application of a microstructure-altering thermal treatment drastically improved their behaviour and their capability for energy absorption. Heat-treated graded lattices exhibited progressive layer collapse and incremental strengthening. Graded and uniform structures absorbed almost the same amount of energy prior to densification, $6.3 \pm 0.2 \mathrm{MJ} / \mathrm{m}^{3}$ and $5.7 \pm 0.2$ $\mathrm{MJ} / \mathrm{m}^{3}$, respectively, but densification occurred at around $7 \%$ lower strain for the graded structures. Several characteristic properties of SLM aluminium lattices, including their effective elastic modulus and Gibson-Ashby coefficients, $C_{1}$ and $\alpha$, were determined; these can form the basis of new design methodologies for superior components in the future.
\end{abstract}

Keywords: selective laser melting, additive manufacture, lattice, mechanical testing, functional grading

\section{Introduction}

Cellular solids have been investigated for some time to determine relationships between aspects of their geometry and their physical properties (thermomechanical, acoustic, crashworthiness, etc.)[1-7]. The motivation for much of this research has been so that they might be used in high-performance,

\footnotetext{
* Corresponding author

Email address: ian.maskery@nottingham.ac.uk (I. Maskery)

Preprint submitted to Elsevier
} 


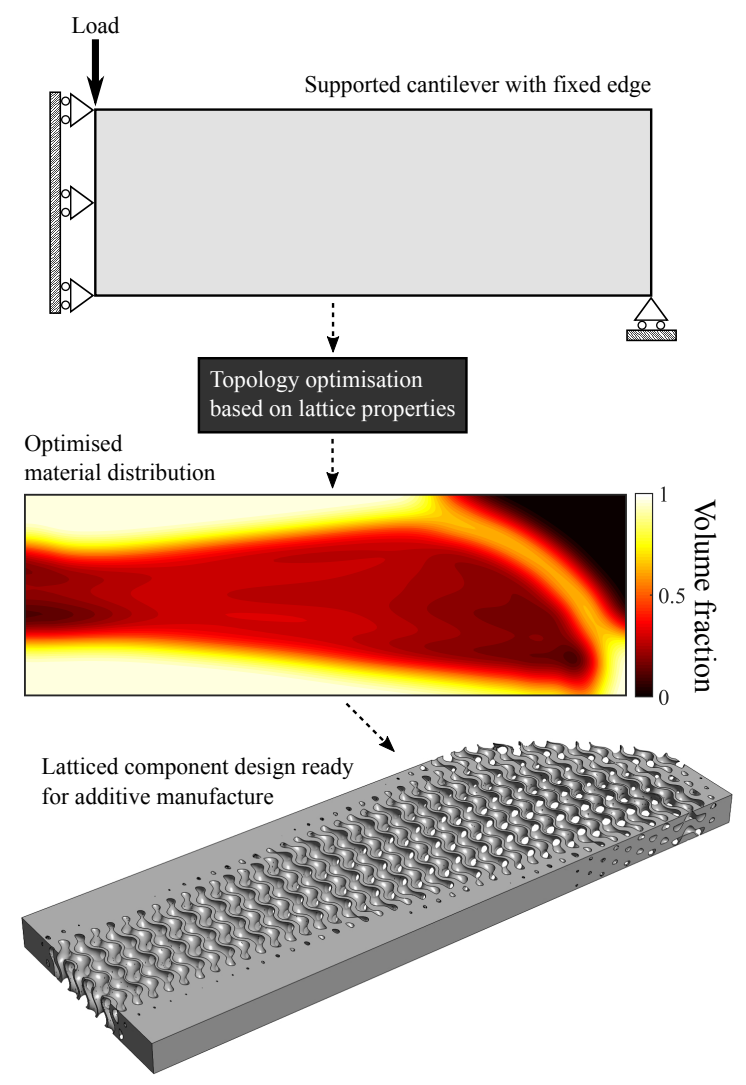

Figure 1: Example of topology optimisation coupled with lattice generation for AM. The example is a simple $2 \mathrm{D}$ cantilever problem. The optimised solution provides the material distribution in the centre of the figure, which is then latticed to provide the structure shown below.

lightweight, applications; for example in simultaneous structural lightweighting, sound dampening and crash energy absorption in motor vehicles and aircraft, with the ultimate aim being to provide high levels of safety and comfort for passengers, whilst minimising resource consumption and the generation of 20 greenhouse gases.

The manufacturing route for metal cellular solids has traditionally been to produce a foam and machine it to the required shape[8], or to stack and bond laminar sheets of periodic structures[9, 10]. Investment casting has also been pursued for this purpose[11], but its effectiveness is limited by the complexity 25 of the structures[12]. These manufacturing routes comprise several processes, are generally costly and materially inefficient, and are unable to yield cellular structures with the complex net shapes required for advanced applications.

With the advent of multi-objective topology optimisation (MTO)[10, 13$15]$ and metal additive manufacturing processes (AM) such as selective laser 
30 melting (SLM), complex designs that feature cellular or latticed regions are now realisable. AM provides the means to produce net-shape lattice structures with almost complete geometric freedom. This control extends to the shape and size of the periodic cells and, therefore, also to the fractions of void and solid material in the structure.

35 Recent advances in lattice design have enabled the creation of structures with spatially varying solid volume fraction[16][17]; these are termed functionally graded lattices. The purpose of this investigation is to examine the performance of functionally graded lattices compared to those of uniform volume fraction, and also to assess SLM aluminium lattices in the context of the pre-existing

40 Gibson-Ashby mechanical behaviour theoretical framework. Investigations of this kind will be crucial in establishing effective component design approaches that combine topology optimisation and lattice structures. One such approach is the combined topology optimisation and error diffusion method described in reference [16]. In that work a topology optimisation algorithm is used to deter5 mine the optimal distribution of material in terms of material density, which is then converted via greyscale representation to a graded lattice by a dithering process. This approach has been demonstrated for a number of structures, such as the cantilever plate illustrated in figure $1[18]$.

Of principal relevance to this work is a paper from the same authors[19]

${ }_{50}$ documenting an investigation into functionally graded lattices made by selective laser sintering (SLS). In that work, the deformation and resulting stress-strain curves of two types of graded and non-graded lattice were analysed, with the graded density structures being found to have much greater potential for energy absorption than their non-graded counterparts. Several important parameters relating the mechanical performance of those lattices to their volume fraction were obtained.

There have been very few reported investigations into graded density structures made by metal AM processes, however, these are more promising than polymer structures in terms of their stiffness, collapse strength and energy ab60 sorption. A graded density Ti-6Al-4V lattice made by electron beam melting (EBM) was studied by Van Grunsven et al.[20] They observed the progressive deformation and collapse of increasingly dense layers in their specimen, noting that this feature might be of use in the biomedical sector (surgical implants, for example) and could offer protection from dynamic loads. Other work on graded 65 metal cellular solids has focussed on foams[21-25] and honeycombs[26, 27], with a result from Brothers and Dunand[22] clearly demonstrating the differences in compressive behaviour between graded and non-graded cellular structures.

In this report, we further our previous work on functionally graded lattices by extending the investigation to metal structures made by SLM. We demon70 strate that an aluminium SLM lattice exhibiting brittle crushing behaviour, and therefore low and non-progressive energy absorption throughout structural collapse, can be modified with a post-manufacture thermal treatment to provide more ideal crushing behaviour, as well as much improved energy absorption. We show that a functionally graded lattice can be used to provide a progressive 75 response to an applied load, whilst absorbing the same amount of energy up to 
structural densification as a non-graded lattice of the same mass. Finally, we empirically determine the Gibson-Ashby parameters $C_{1}$ and $\alpha$ for the examined metal lattices structures. These define the relationships between lattice volume fraction, effective modulus and densification strain, and are required for the design of advanced lightweight components incorporating lattice structures and topology optimisation.

\subsection{The Gibson-Ashby model of lattice deformation}

The most significant theoretical work regarding the deformation and mechanical properties of cellular solids is that of Gibson and Ashby[2]. The models

85 they developed were originally applied to experimental results from honeycomb and foam structures, but they have more recently been used in the investigation of lattices made by $\mathrm{AM}[28-31]$.

Gibson and Ashby[2] treated foams as arrays of connected beams and provided a series of equations relating their mechanical properties to their volume fraction (or relative density). The current work makes use of two Gibson-Ashby equations; these are

$$
\begin{aligned}
& \frac{E_{\text {latt. }}}{E_{\text {sol. }}}=C_{1}\left(\frac{\rho_{\text {latt. }}}{\rho_{\text {sol. }}}\right)^{n}, \\
& \varepsilon_{D}=1-\alpha\left(\frac{\rho_{\text {latt. }}}{\rho_{\text {sol. }}}\right),
\end{aligned}
$$

where the corresponding physical properties are provided in table 1 . The parameters $n, C_{1}$ and $\alpha$ were determined to be $\sim 2,0.1-4.0$ and $1.4-2.0$, 90 respectively, through comparison with experimental data[1, 2]. Equations 1a and $1 \mathrm{~b}$ have been shown to describe well the mechanical properties of polymer and metal foams with a range of relative densities[1][2], but importantly, Gibson and Ashby indicated that, "At large densities $\left(\rho^{*}>0.3\right)$ the beam-bending concept breaks down ... Above this density the material is better thought of 95 as a solid with holes in it, not a foam[2]." This is because struts in higher density cellular solids tend to have low aspect ratio, and therefore yield under axial strain, while the more slender struts of low density cellular solids undergo bending and their deformation is adequately described by the beam theory underpinning the formulae of Gibson and Ashby. The symbol $E^{*}$ is often used for the relative elastic modulus of a lattice; this is equal to $E_{l a t t .} / E_{\text {sol. }}$. Similarly, $\rho^{*}$, the relative density of a lattice, is equal to $\rho_{\text {latt. }} / \rho_{\text {sol. }}$.

Gibson and Ashby[2] provided schematic stress-strain curves for foams under compressive stress, as reproduced here in figure 2. They show that structural collapse exhibits three distinct regions of stress-strain behaviour. The first is linear elasticity characterised by the modulus, $E_{\text {latt. }}$. The deformation in this region is principally due to the bending of cellular struts. The stress-strain behaviour in the second region depends on the properties of the material of which the structure is composed. If the material exhibits plastic yielding there will be a long collapse plateau at a stress of $\sigma_{p l}$ latt. This can be attributed to the 
Table 1: Physical and mechanical properties used in the description of lattices under compression.

\begin{tabular}{ll} 
Notation & Physical or mechanical property \\
\hline$\rho_{\text {latt. }}$ & Density of the lattice \\
$\rho_{\text {sol. }}$ & Density of the lattice strut material (e.g. aluminium) \\
$\rho^{*}$ & Relative density of the lattice, equal to $\rho_{\text {latt. }} / \rho_{\text {sol. }}$ \\
\hline$E_{\text {latt. }}$ & Elastic modulus of the lattice \\
$E_{\text {sol. }}$ & Elastic modulus of the lattice strut material \\
$E^{*}$ & Relative elastic modulus of the lattice, equal to $E_{\text {latt. }} / E_{\text {sol. }}$ \\
\hline$\sigma_{\text {latt. }}$ & Effective stress of the lattice under deformation \\
$\sigma_{\text {pl. latt. }}$ & Plateau strength of the lattice (for plastic materials) \\
$\sigma_{c r . \text { latt. }}$ & Crushing strength of the lattice $($ for brittle materials) \\
\hline$\varepsilon_{l a t t .}$ & Effective strain of the lattice under deformation \\
$\varepsilon_{D}$ & Densification strain of the lattice \\
\hline$W_{V}$ & Energy absorbed per unit volume under deformation \\
$W_{V_{D}}$ & Total energy absorbed per unit volume up to densification \\
\hline$n$ & Gibson-Ashby parameter relating $E^{*}$ to $\rho^{*}-$ see equation $1 \mathrm{a}$ \\
$C_{1}$ & Gibson-Ashby parameter relating $E^{*}$ to $\rho^{*}-$ see equation $1 \mathrm{a}$ \\
$\alpha$ & Gibson-Ashby parameter relating $\varepsilon_{D}$ to $\rho^{*}-$ see equation $1 \mathrm{~b}$ \\
\hline
\end{tabular}

creation of plastic hinges at the intersections, or nodes, of the cellular struts. If the material is more brittle, this intermediate region will show fluctuations due to brittle fracture in individual cells. This will occur at a stress of $\sigma_{c r}$ latt. . It is the long collapse plateau and its associated energy absorption that make lattice structures so compelling for use in impact absorbing applications[1, 4, 32, 33].

115 Finally, at a strain of $\varepsilon_{D}$, the structure enters a densification regime, where the struts of collapsed cells are forced into contact leading to a significant increase in stiffness. For energy absorbing foams in protective applications (e.g. packaging or personal protective equipment), $\varepsilon_{D}$ represents a practical limit to the usefulness of the structure, since, above this level of strain, energy is absorbed at the expense of increasing transmitted stress.

In this work $\varepsilon_{D}$ were determined using the energy efficiency method outlined by Miltz and Ramon[34] and Li et al.[35] The efficiency of a cellular structure under deformation, $\eta\left(\varepsilon_{\text {latt. }}\right)$, is the ratio of absorbed energy up to a given stress divided by that stress; it is therefore defined by

$$
\eta\left(\varepsilon_{\text {latt. }}\right)=\sigma_{\text {latt. }}\left(\varepsilon_{\text {latt. }}\right)^{-1} \int_{0}^{\varepsilon_{\text {latt. }}} \sigma_{\text {latt. }}\left(\varepsilon_{\text {latt. }}\right) \mathrm{d} \varepsilon_{\text {latt. }},
$$

where $\sigma_{\text {latt. }}$ and $\varepsilon_{\text {latt. }}$ are the effective stress and strain of the lattice structure, respectively. $\varepsilon_{D}$ is then the strain at which $\eta\left(\varepsilon_{\text {latt. }}\right)$ reaches a maximum on the efficiency-stain curve, that is, the strain which meets the condition

$$
\frac{\mathrm{d} \eta\left(\varepsilon_{\text {latt. }}\right)}{\mathrm{d} \varepsilon_{\text {latt. }}}=0 .
$$



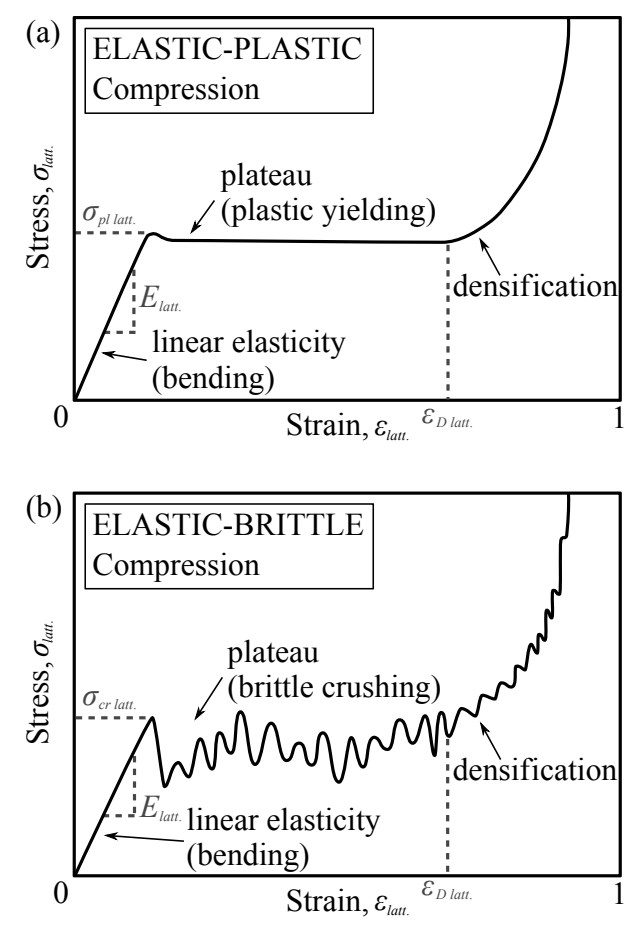

Figure 2: Schematic compressive stress-strain curves for lattices of (a) elastic-plastic and (b) elastic-brittle materials. The noteworthy features, $E_{l a t t .}, \sigma_{p l}$ latt., $\sigma_{c r}$ latt. and $\varepsilon_{D}$ are as described in table 1 . These are reproductions of similar schematics for foams by Gibson and Ashby[2]. 


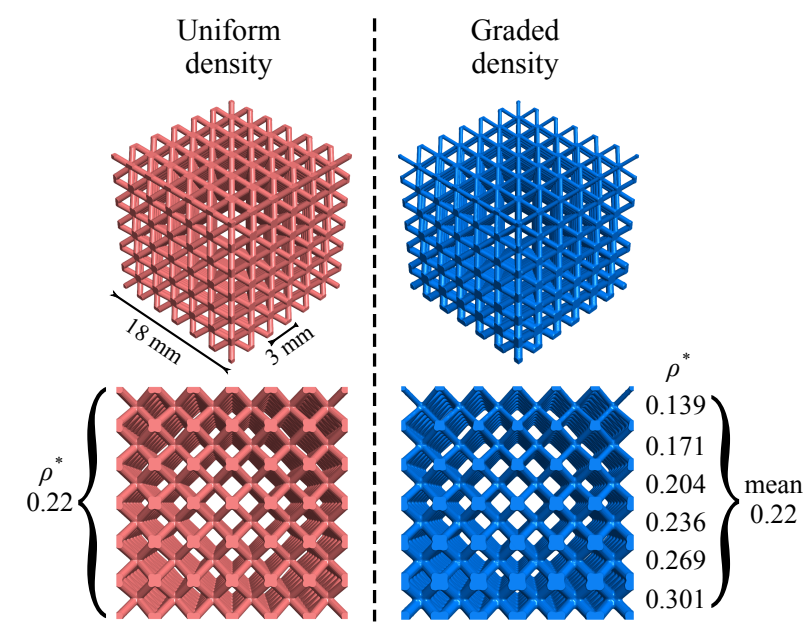

Figure 3: CAD representations of uniform (left) and graded density (right) lattice structures.

\section{Experimental details}

\subsection{Fabrication of the lattice specimens}

130 minium alloy Al-Si10-Mg using a Renishaw AM250 SLM machine. The feedstock powder was provided by LPW Technology; the chemical composition of its principal elements was $\mathrm{Al} 88.9 \mathrm{wt} \%$, Si $10.7 \mathrm{wt} \%, \mathrm{Mg} 0.5 \mathrm{wt} \%$, and the powder particles ranged in size from $15 \mu \mathrm{m}$ to $110 \mu \mathrm{m}[36]$. The Renishaw AM250 maparts with a hatch spacing of $130 \mu \mathrm{m}$, a point distance of $80 \mu \mathrm{m}$ and an exposure time of $140 \mu \mathrm{s}$. During SLM processing, the $\mathrm{O}_{2}$ concentration in the atmosphere was kept below $500 \mathrm{ppm}$ using a positive pressure of $\mathrm{Ar}$ gas. The Al-Si10-Mg platform was held at $180{ }^{\circ} \mathrm{C}$ during specimen production. The individual cells in the lattice structures were $(3 \times 3 \times 3) \mathrm{mm}$ in size, thus providing lattices containing a $(6 \times 6 \times 6)$ array of cells.

The lattices comprised cells of the body-centred-cubic (BCC) type. The $\mathrm{BCC}$ cell is a 3D intersection of struts angled at $45^{\circ}$ to vertical, as illustrated in figure 3. Figure 3 also highlights the geometrical features of the two forms of BCC lattice examined in this study; those of uniform relative density (nongraded) and those in which the relative density of a region is determined by its position in the structure. In this work the relative density of the graded

150 structures was described by a linearly decreasing function of the height; that is, the lattices had thicker struts (and therefore a higher relative density) at their bases, and thinner struts (and therefore a lower relative density) at their tops. This can be seen in figure 3 and the side-on optical micrographs of the manufactured specimens in figure 4 . To ensure that the mechanical performance 


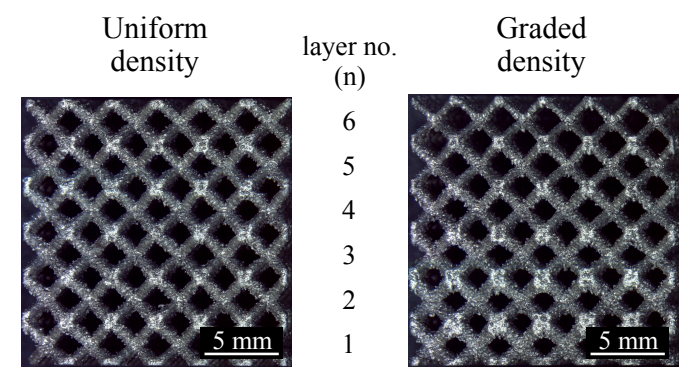

Figure 4: Optical micrographs of uniform (left) and graded density (right) lattice structures.

of the graded and non-graded structures were compared on an equal-mass basis, the average relative density of the two lattice types was identical; the value being set at 0.22 . For the graded structures, this was achieved by designing the relative densities of each the six cellular layers to be $0.301,0.269,0.2360 .204$, 0.171 and 0.139 . The corresponding strut thicknesses of the graded structure ranged from $0.91 \mathrm{~mm}$ to $0.42 \mathrm{~mm}$, with abrupt, incremental changes at the layer boundaries. An alternative grading approach is to continuously vary the intracellular strut thickness, providing a smoother gradation to the structural density profile. The latter design approach is more challenging, but is no less achievable with AM processes. The relative densities of all of the examined lattices were found to be in good agreement with the designed mean value of 0.22 , and the strut thicknesses, obtained from optical micrographs, also agreed with those of the CAD models. This evidence, in a addition to a previous result[37] showing porosity less that $0.1 \%$ in solid Al-Si10-Mg produced using the same Renishaw AM250 SLM machine, indicates that the geometries of the manufactured lattice 170 specimens corresponded well with the intended designs.

\subsection{Post-manufacture heat treatment}

Based on previous research[38], the graded and non-graded lattice specimens were subject to a post-manufacture heat treatment. The aim was to improve their ductility, and therefore their suitability for the energy-absorbing applications which motivate much research into cellular solids. The heat treatment comprised a solution treatment for 1 hour at $520^{\circ} \mathrm{C}$ followed by a water quench and artificial ageing for 6 hours at $160{ }^{\circ} \mathrm{C}$. At both of the heating steps in this process, the samples were introduced directly into a pre-heated furnace with an air atmosphere. Such a treatment was found by Aboulkhair et al.[38] to modify the microstructure of SLM Al-Si10-Mg and reduce its hardness by nearly $30 \%$ from the as-built condition. The softening demonstrated by Aboulkhair et al. is in contrast to the hardening effect of this type of heat treatment on conventionally produced material; the reasons for this are discussed in detail in reference [38]. The effect of the post-manufacture heat treatment on the SLM Al-Si10-Mg microstructure is shown in figure 5; the as-built SLM material, with its characteristic melt pools and fine microstructure, is transformed into a coarser-grained material, where the Si has agglomerated into equiaxed particles. 

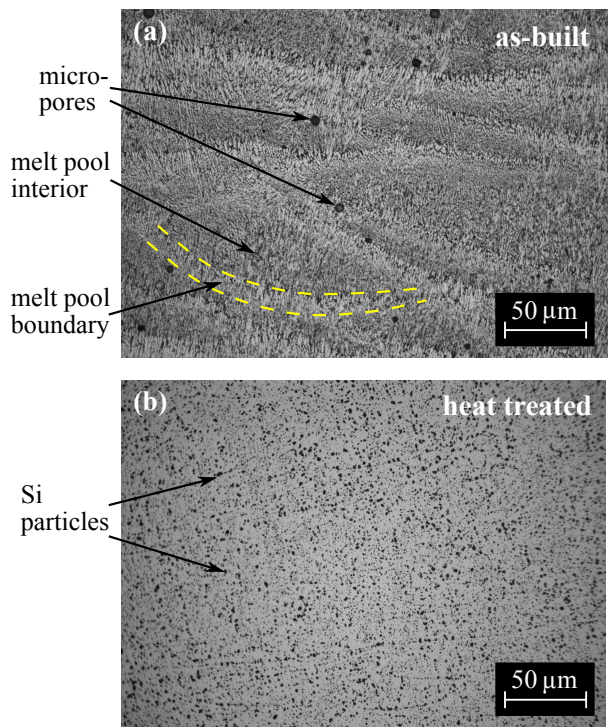

Figure 5: SEM micrographs of as-built (a) and heat treated (b) Al-Si10-Mg produced by SLM. Note that several micropores can be seen in (a) as dark circles; these should not be mistaken for the large equiaxed Si particles that feature in (b).

Representative tensile stress-strain data from as-built and heat treated SLM $\mathrm{Al}-\mathrm{Si} 10-\mathrm{Mg}$ dog bone specimens are presented in figure 6 . The tensile specimens 190 were designed following ASTM standard E8/E8M[39]. They had $45 \mathrm{~mm}$ gauge length, $9 \mathrm{~mm}$ gauge diameter, and were manufactured with their long axes perpendicular to the SLM build platform[40]. They were manufactured in net shape on a separate build platform from that used in the production of the lattice structures. Three dog bone specimens in each condition, as-built and heat treated, were tested, but for clarity only one data set from each is presented in the figure. Mechanical properties determined from the stress-strain curves are provided in table 2 . From these we can identify two important features which we expect to influence the deformation behaviour of the lattice structures. First, the as-built material shows much lower failure strain than the heat treated material; the mean values of failure strain from three repeat tests are $(1.4 \pm 0.3) \%$ and $(3.9 \pm 0.5) \%$, respectively, for as-built and heat treated material. Second, the heat treatment caused a lowering of the ultimate tensile strength from $330 \pm 10$ $\mathrm{MPa}$ to $292 \pm 4 \mathrm{MPa}$, a reduction of $(12 \pm 3) \%$. The elastic modulus of the material was unaffected by the application of the heat treatment.

\subsection{Mechanical testing}

Uniaxial compression tests of the lattice structures were conducted using an Instron 5969 universal testing machine equipped with a $50 \mathrm{kN}$ load cell. The compressive displacement was applied at a rate of $0.03 \mathrm{~mm} / \mathrm{s}$. Strain data were collected using a linear variable differential transformer (LVDT), while the de- 


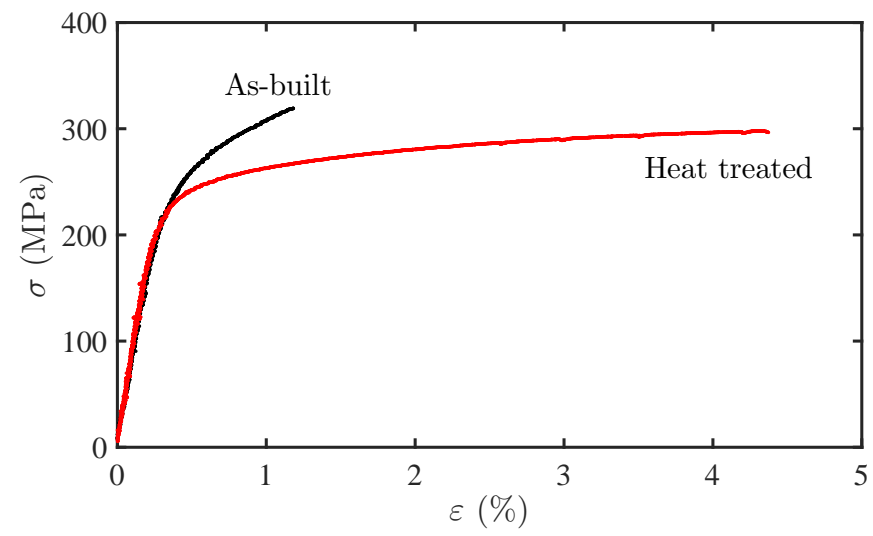

Figure 6: Representative tensile stress-strain curves of as-built and heat treated SLM Al-Si10Mg dog bone specimens.

Table 2: Elastic modulus, ultimate tensile strength and failure strain of as-built and heat treated SLM Al-Si10-Mg.

\begin{tabular}{lll} 
& As-built & Heat treated \\
\hline$E_{\text {sol. }}(\mathrm{GPa})$ & $81 \pm 2$ & $80 \pm 2$ \\
$\sigma_{U T S}$ sol. $(\mathrm{MPa})$ & $330 \pm 10$ & $292 \pm 4$ \\
$\varepsilon_{U T S \text { sol. }}(\%)$ & $1.4 \pm 0.3$ & $3.9 \pm 0.5$ \\
\hline
\end{tabular}

formation of each specimen was simultaneously recorded by video camera at a rate of 50 frames per second. Frames extracted from these videos were then correlated with features in the associated stress-strain curves, providing information about the failure modes of the graded and non-graded lattice structures.

\section{Results and discussion}

\subsection{Deformation of lattices with as-built microstructure}

Data from an aluminium SLM lattice tested in its as-built condition are presented in figure 7 . The data, along with the inset video frames, show a significant structural collapse at around $9 \%$ strain. This collapse, in which $95 \%$ of the structure's strength is lost, follows an initial period of linear elasticity and non-linear behaviour where the cell struts begin to bend under compressive loading. After slight bending some of the struts experience brittle fracture[41], in this case resulting in a diagonal $\left(45^{\circ}\right)$ shear band of the kind previously documented for similar lattice structures[42, 43]. The structure is subsequently weakened, and does not recover its pre-collapse strength even as the strain increases up to $50 \%$, at which point densification, in which some of the remaining cell struts are forced into contact, begins to play a role in reestablishing the lost strength. This crushing behaviour is in good agreement with that observed for aluminium SLM lattices in a previous investigation[43]. 


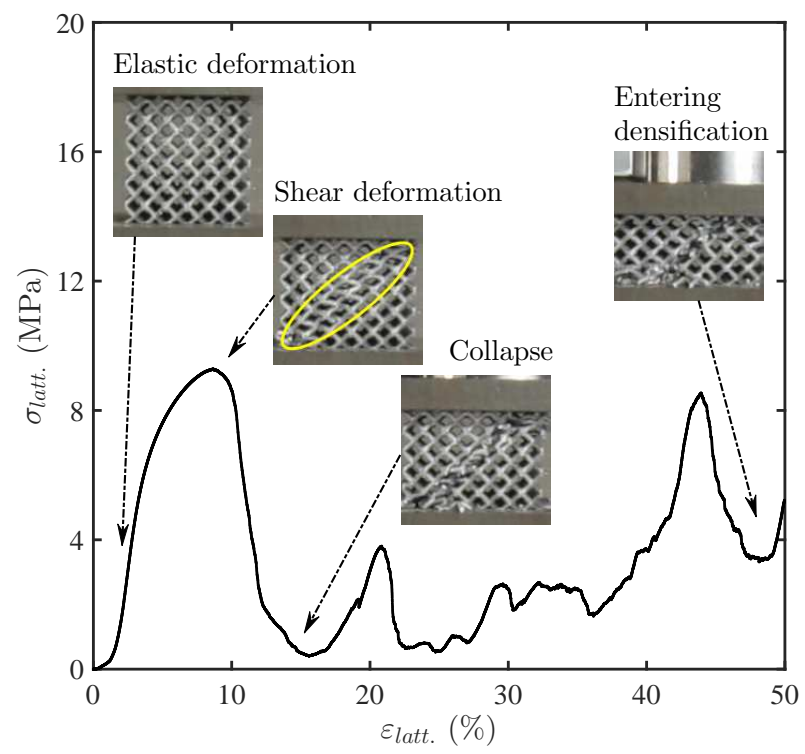

Figure 7: Compressive stress-strain curve of an as-built uniformly dense aluminium lattice. Inset are video frames showing the condition of the lattice structure at several stages during compression.

Fracture surfaces from an as-built lattice shown in figure 8 provide a more complete picture of the fracture mechanism. Fracture occurs almost exclusively at the lattice nodes (figure 8(a)), and these show a combination of pore opening (figures 8(b) and 8(c)), and ductile dimpling associated with both tensile and shear loading (figures 8(b)-(f)). It was suggested in a previous study on SLM aluminium lattices that nodal cracking of this kind originates at the sites of 235 internal pores[43].

\subsection{Heat treated lattices}

Lattices subject to post-manufacture heat treatment underwent a collapse process that, like the as-built lattice, featured a diagonal shear band occurring at around $10 \%$ strain - see the video frames in figure 9 and the stress-strain curves

240 in figure 10. Unlike the as-built lattice however, the reduction in strength was smaller, at just $75 \%$, and the heat treated lattices regained their pre-collapse strength upon the compressive strain increasing to around $20 \%$. This strength restoration was seen for all three tested specimens, and their deformation behaviour in the $0 \%-25 \%$ strain region was quite consistent. As before, for the 245 sake of clarity, only one data set from three repeat tests of each specimen type is shown in figure 10.

Examining figure 10 further, it can be seen that following the initial collapse and re-strengthening, the heat treated lattices underwent further, but less severe, weakening in the region of $25 \%-35 \%$ strain. The behaviour of the three 


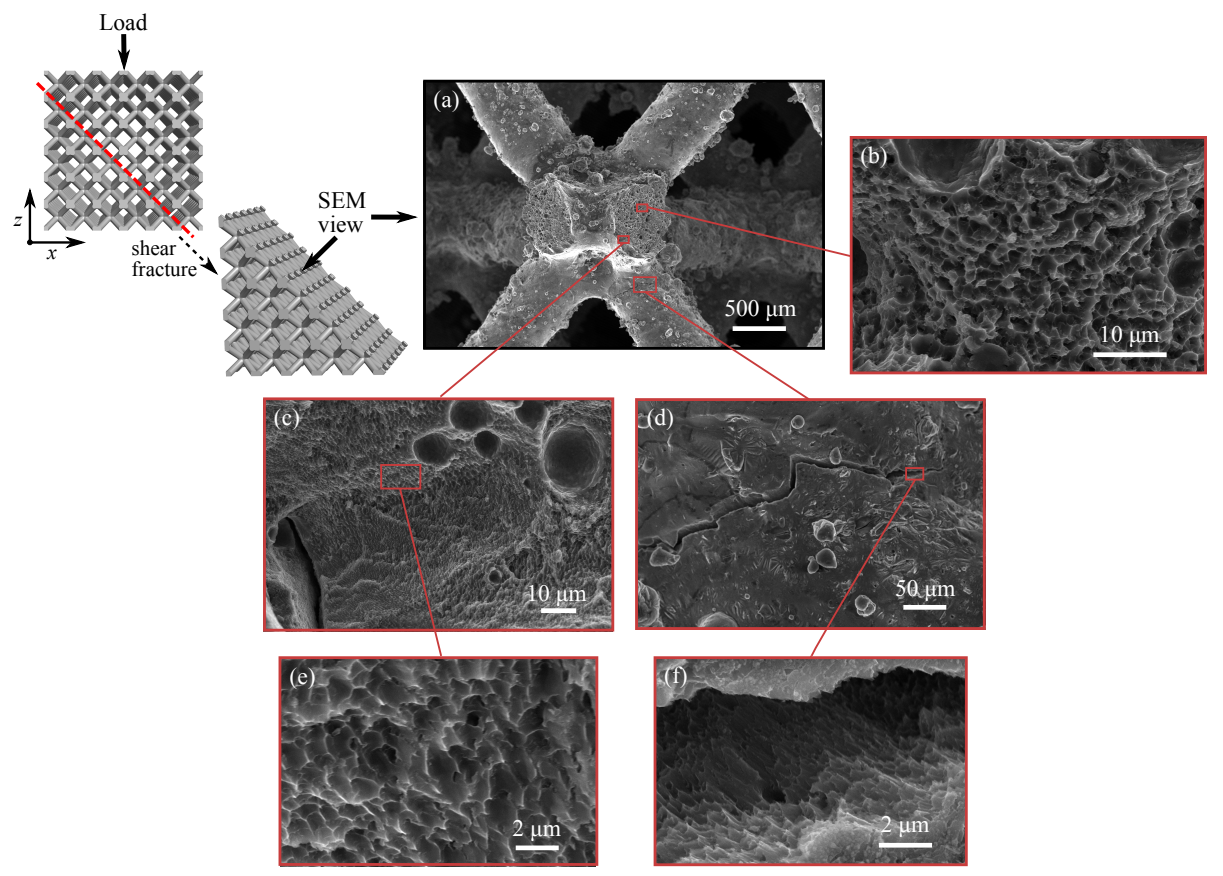

Figure 8: Secondary electron SEM micrographs of a strut fracture surface from an as-built lattice specimen. The schematic (upper left) illustrates the lattice specimen orientation and loading direction with respect to the SEM micrographs.
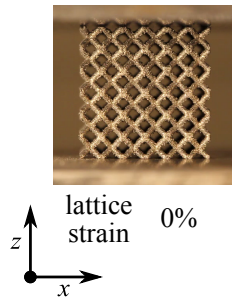

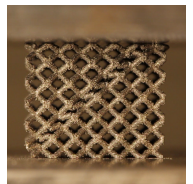

$12 \%$

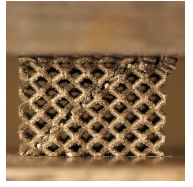

$23 \%$

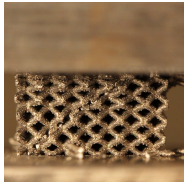

$41 \%$

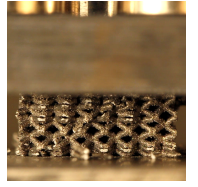

$56 \%$

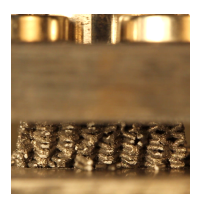

$68 \%$

Figure 9: Video frames from the compression of a heat-treated graded density aluminium lattice structure. 


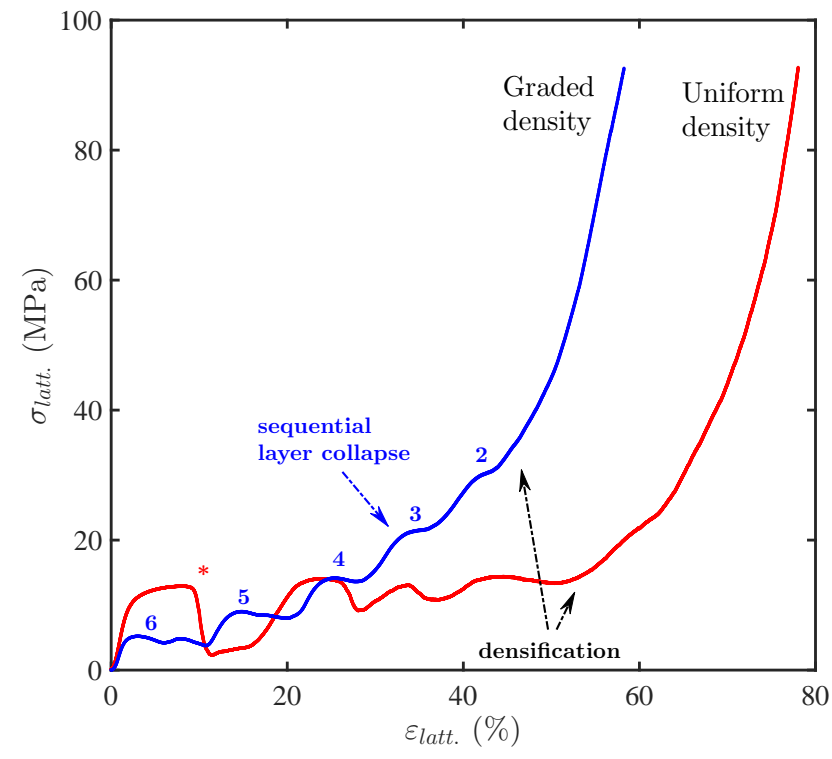

Figure 10: Compressive stress-strain curves of heat-treated aluminium lattices structures. The numbers $6,5,4,3,2$ indicate the collapse of successive layers of the graded density structures, while $*$ indicates a diagonal shear failure of the uniformly dense structures.

specimens in this region showed less consistency than during the initial collapse, but in all cases the strength was again restored to around the pre-collapse level. These features in the stress-strain curves were likely due to the structural collapse and subsequent densification of a whole cellular layer either at the top or base of the specimen (i.e. layer 1 or layer 6 in figure 4). In these layers the unattached 'free' struts are able to deform more readily in the directions orthogonal to compressive loading than the struts toward the center of the structure. In general, at stress levels above $35 \%$ the heat treated lattices exhibited stress-strain behaviour more akin to the ideal behaviour of cellular solids. They featured relatively flat plateaux followed by large increases in stress at the onset of densification.

The difference in deformation behaviour between heat treated and as-built SLM aluminium lattices can be explained by the drastically different microstructure and resistance to fracture of the lattice strut material. Despite the existence of the structural collapse and diagonal shear band at close to the same level of strain for both as-built and heat treated lattices, there is evidence that the fracture micromechanisms are quite different. This is shown in the heat treated fracture surfaces in figure 11, which show much coarser features than are present in the corresponding images from an as-built lattice (figure 8). This can be correlated with the alteration in the size and distribution of alloying 270 inclusions brought about by the heat treatment (figure 5). The ductile dimples seen in figures 11(a) and 11(c) are approximately twice as large as those seen in 

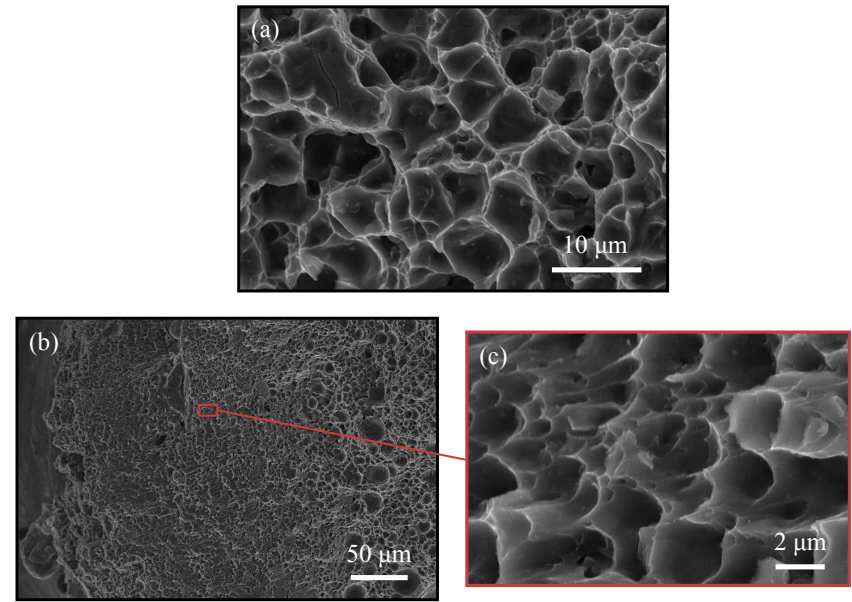

Figure 11: Secondary electron SEM micrographs of a strut fracture surface from a heat treated lattice specimen.

Table 3: Mechanical properties of heat treated uniform density lattice structures.

\begin{tabular}{ll}
\hline$E_{\text {latt. }}(\mathrm{GPa})$ & $0.64 \pm 0.02$ \\
$E^{*} \times 10^{-3}$ & $8.0 \pm 0.3$ \\
$C_{1}$ & $0.166 \pm 0.006$ \\
\hline
\end{tabular}

comparable images from as-built fracture surfaces (figures 8(b) and 8(e)).

From the stress-strain curves of heat treated uniform density lattices can be extracted their modulus, $E_{\text {latt. }}$. This, along with $E^{*}$ (which is $E_{\text {latt. }}$ normalised by the modulus of the bulk material, $80 \pm 2 \mathrm{GPa}$ ), is given in table 3 . Using $E^{*}$ in equation 1a yields the Gibson-Ashby prefactor $C_{1}$, which has the value $0.166 \pm 0.006$. This is toward the lower end of the range given by Gibson and Ashby[2], which was $0.1-4.0$. The value of $C_{1}$ determined here agrees well with the values of 0.17 and 0.19 reported for two types of Ti-6Al- $4 \mathrm{~V}$ lattice by Yan et al.[44], and is somewhat lower than the value of $0.44 \pm 0.01$ determined for the same BCC lattice structure made in a polymer by SLS[19]. It is worth noting, however, that the deduction of $C_{1}$ is here based on the assumption that the lattices follow an $E^{*} \propto \rho^{* 2}$ proportionality. It has been shown elsewhere[4446] that the experimentally determined $\rho^{n}$ exponent can take values between 1.64 and 2.84, depending on the lattice cell type and the presence of surface roughness and residual stresses resulting from the manufacturing process.

\subsection{Graded density lattices}

In contrast to the uniformly dense lattice structures, those with graded density showed novel deformation behaviour, as seen in figure 12. Beginning with the layers of lowest relative density at the tops of the specimens, the lattices collapsed in a layer-by-layer sequence, with each layer collapse possessing the 


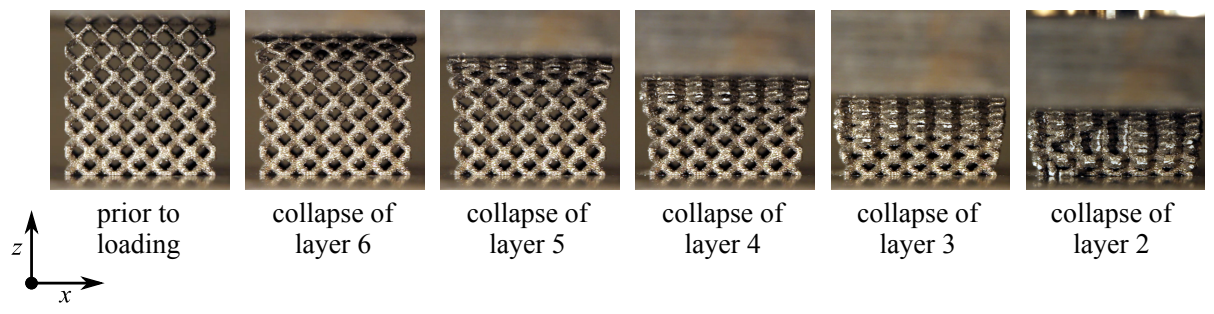

Figure 12: Video frames from the compression of a heat-treated graded density aluminium lattice structure.

Table 4: Parameters associated with lattice densification under compressive deformation.

\begin{tabular}{lll} 
& Uniform density & Graded density \\
\hline$\varepsilon_{D}(\%)$ & $53 \pm 2$ & $45.6 \pm 0.3$ \\
$\alpha$ & $2.12 \pm 0.08$ & $2.47 \pm 0.01$ \\
\hline
\end{tabular}

linear stress rise and plateau which are characteristic of ideal cellular solids. Significantly, there is an absence of the diagonal shear band commonly seen in the compressive collapse of the BCC structure. The density gradient therefore eliminates the large drop in strength associated with the shear collapse, and also has implications for the energy absorption of the lattices, as discussed in section 3.4. The sequential layer collapse can be seen in the step-like features of the stress-strain curves in figure 10, where numerical notation is used to indicate the collapse of the layers identified in figure 4. This result is consistent Ti-6Al-4V lattice examined by van Grunsven et al.[20]

The sequential layer collapse of the graded lattices continued up to the densification of the final layer, at which point the stress was seen to rise drastically, much like the densification of uniformly dense structures. The onset of densification occurred at a lower level of strain for the graded structures than the uniformly dense structures; the densification strains, $\varepsilon_{D}$, were $(45.6 \pm 0.3) \%$ and $(53 \pm 2) \%$, respectively. From these $\varepsilon_{D}$ values can be determined a critical parameter related to the performance of uniform and graded density lattices. This is $\alpha$ from equation $1 \mathrm{~b}$, and is provided in table 4 . The determined values 310 for $\alpha$ lie above the range of $1.4-2.0$ given by Gibson and Ashby[2], indicating that the onset of densification occurred at lower strains than might be predicted for structures of this volume fraction. However, there is reasonable agreement with an $\alpha$ value of $2.449 \pm 0.002$ previously reported for a BCC lattice structure made by SLS[19].

315 After densification the graded density lattice specimens were reduced to a compacted, but largely intact, structure (figure 13(a)), microscopic examination of which (figure 13(b)) revealed that pronounced strut bending had occurred in the topmost cellular layers, while nodal cracking, of the kind seen in uniformly dense lattices, occurred in the layers below that comprised thicker struts.

Figure 13 raises the issue of the comparability of $\varepsilon_{D}$ and $\alpha$ values for the 

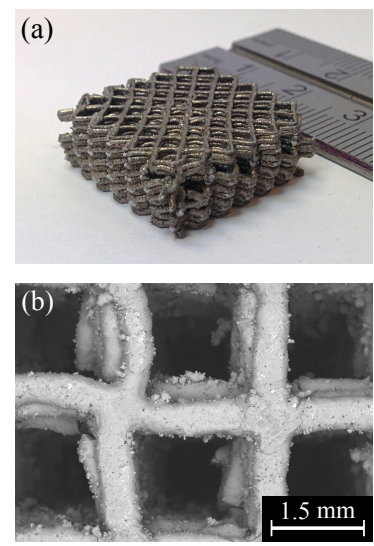

Figure 13: Photograph (a) and SEM micrograph (b) of a graded density heat treated lattice specimen after compressive testing beyond the densification strain.

examined uniform and graded density lattices. The deformation mechanism in the uniform density lattices (in this case, strut bending followed by nodal fracture) is unchanging throughout the compression process up to the point of densification; this is because all of the struts are of the same thickness, and 325 therefore respond equivalently to the applied load. On the other hand, deformation in the graded lattices transitions gradually from a bending and plastic collapse mode in the low density region at the top of the structure (where few of the struts exhibit fracturing), to one in which nodal fracture occurs. For this reason, values of $\varepsilon_{D}$ and $\alpha$ are likely to be highly sensitive to the nature of the strut thickness gradient in a given lattice structure, and the associated transition, or transitions, between failure types throughout deformation.

\subsection{Energy absorption under compressive deformation}

$\eta\left(\varepsilon_{\text {latt. }}\right)$ for graded and non-graded lattices are provided in the inset of figure 14; the positions of their maxima prior to the decreasing trends after densification provide the values of $\varepsilon_{D}$. Shown in the main plot of figure 14 are the cumulative energy absorption per unit volume, $W_{V}$, as a function of lattice strain. Prior to densification $W_{V}\left(\varepsilon_{\text {latt. }}\right)$ is almost linear for the uniform density lattices, with a feature of reduced energy absorption at around $10 \%$ lattice strain due to the structural collapse discussed previously. $W_{V}\left(\varepsilon_{\text {latt. }}\right)$ for the graded 340 density structures exhibited very different behaviour, increasing geometrically with $W_{V}\left(\varepsilon_{\text {latt. }}\right)$ roughly proportional to $\varepsilon_{\text {latt. }}{ }^{2}$. Similar behaviour was observed for BCC lattice structures made by SLS in a polymeric material[19], but in that case $W_{V}\left(\varepsilon_{\text {latt. }}\right)$ was found to be roughly proportional to $\varepsilon_{\text {latt. }}{ }^{3}$.

It is worth highlighting that, while the ideally linear energy absorption of the uniform lattices is noticeably impaired by the occurrence of the diagonal shear band, no such impairment exists for the graded density lattice. The density grading, therefore, provides the structure a more predictable deformation 


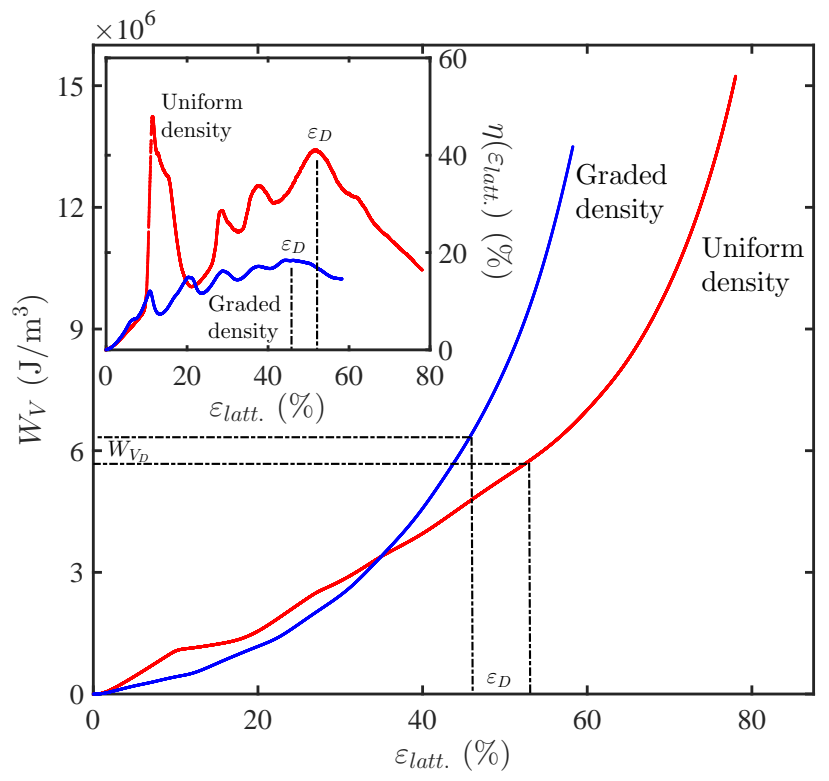

Figure 14: Normalised energy absorption per unit volume and (inset) efficiency of uniform density and graded density lattices under compressive deformation.

and energy absorption profile, making it much more attractive for designers of energy absorbing latticed components. This result also suggests that more sophisticated density gradients or patterns (other than the linear density variation examined here) could be employed to eliminate undesirable failure modes in lattice structures, whilst retaining the benefit of high specific energy absorption.

The total energies absorbed by uniform and graded density lattice structures prior to densification were $5.7 \pm 0.2 \mathrm{MJ} / \mathrm{m}^{3}$ and $6.3 \pm 0.2 \mathrm{MJ} / \mathrm{m}^{3}$, respectively. The close agreement of these values is in contrast to a previous result in which graded density structures were found to absorb significantly more energy under compressive deformation than uniform structures of the same volume fraction[19]. Previous work on aluminium foams with similar volume fractions 360 to the lattices studied here have put their energy absorption at between 3.1 $\mathrm{MJ} / \mathrm{m}^{3}[47]$ (based on finite element simulations) and $\sim 4.5 \mathrm{MJ} / \mathrm{m}^{3}[48]$ (from an investigation into dynamic crushing behaviour). Sources of comparison in which the volume fractions of the examined structures allow direct comparison are scarce, but our $W_{V_{D}}$ values are in keeping with trends observed for Alcan and Alporas foams[49], and with foams of various kinds summarised by Ashby et al. [1].

Finally, in figure 15 is presented $W_{V}$ normalised by the modulus of the lattice strut material, $E_{\text {sol. }}$. This is plotted against the effective stress of the lattice structure, $\sigma_{\text {latt. }}$, equally normalised. This form of energy absorption diagram 370 was used by Maiti et al., and Gibson and Ashby in their discussions of cellular 


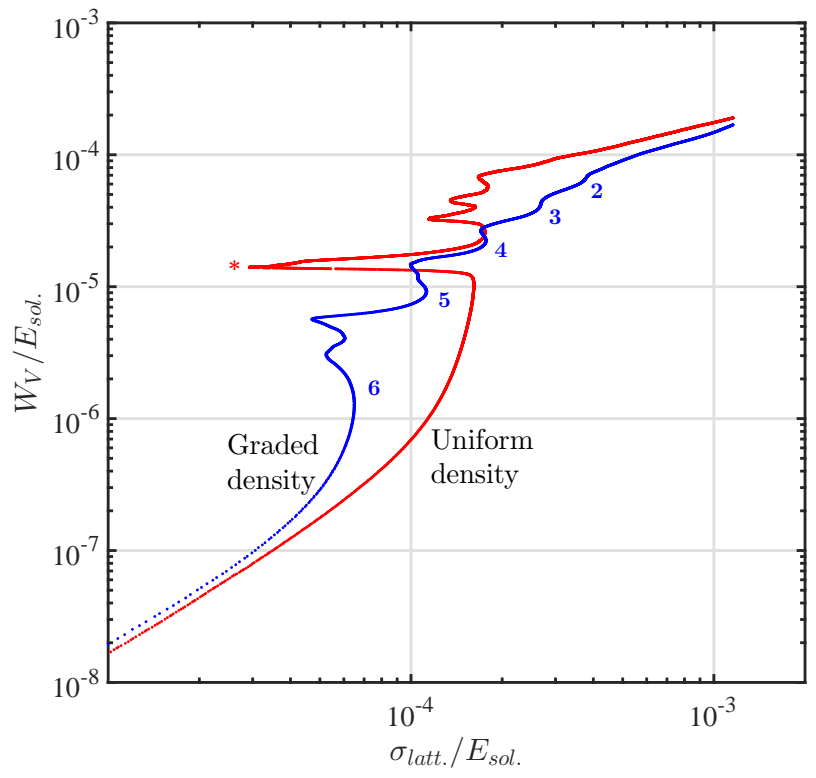

Figure 15: Normalised energy absorption of heat-treated aluminium lattices structures. The numbers $6,5,4,3,2$ indicate the collapse of successive layers of the graded density structures, while $*$ indicates a diagonal shear failure of the uniformly dense structures.

solids $[2,50]$. When data from several cellular solids and several testing conditions are plotted together, this diagram is useful in allowing a designer to choose the one structure or material most suited to a given application. For conventional, non-graded, cellular solids this would be the one which absorbed the most energy up to the maximum stress allowed for that application; for example, the stress that would cause damage to a package (in the case of packaging materials) or harm to a person (in the case of personal protection equipment). For functionally graded structures, the selection criteria can be more particular, with the designer having the freedom to consider the deceleration profile or jerk, the rate of energy absorption, as well as peak stress.

\section{Conclusions}

We have described the first experimental investigation into the crushing behaviour of functionally graded SLM Al-Si10-Mg lattices under quasistatic loading. A previous result[38] indicated that a heat treatment might improve the ductility of the SLM base material and the suitability of these lattices for energy absorbing applications. This was seen to be the case, as evidenced by the increased failure strain of heat treated dog bone specimens, and also the ability of heat treated lattices to recover their strength after a partial collapse and absorb energy in an more ideal manner up to structural densification. This demonstrates that SLM, coupled with a straightforward post-manufacturing 
process, can be used to create lightweight energy-absorbing structures in an aluminium alloy of high industrial relevance.

Graded lattices underwent collapse in a layer-by-layer sequence; progressing from regions of low to high density. The associated stress-strain curves comprised a series of step-like features, where the collapse and densification of each layer were identifiable. This incremental strengthening, and the increasing rate of energy absorption up to densification, make graded structures particularly attractive for advanced applications, as they enable a progressive mechanical response to a static or dynamic load to be achieved by selecting the appropriate material distribution. Density grading also prohibited the occurrence of a diagonal shear band, making the deformation and energy absorption profiles of graded density lattices more predictable than uniform lattices of the same mean density. With these results in mind, the determination of two Gibson-Ashby prefactors, $C_{1}$ and $\alpha$, for the SLM Al-Si10-Mg BCC lattice is a significant result of this investigation. These values can be used to establish the relationships between lattice geometry and performance that are required for lattice generation to be combined with topology optimisation successfully.

There is, however, a limitation of the Gibson-Ashby based analysis as applied to graded density structures, and it is highlighted in this study. The model does 410 not explicitly deal with transitions between lattice failure types (e.g. thin strut bending to nodal cracking) within the same structure, and therefore its ability to predict useful properties such as $\varepsilon_{D}$ and $W_{V_{D}}$ for graded structures may be impaired, at least until sufficient empirical evidence is collected from lattices of different relative densities, cell types and density gradients. Designers of functionally graded lattice components are therefore advised to also consider finite element models in their analyses. A combined empirical and numerical approach will ultimately provide designs for a new generation of lightweight, functionally graded lattice components with highly tailorable properties.

\section{Acknowledgments}

Funding was provided by the EPSRC. Thanks to Mark East, Mark Hardy, Joe White, Jason Greaves and Tom Buss.

[1] M. Ashby, A. Evans, N. Fleck, L. Gibson, J. Hutchinson, and H. Wadley, Metal Foams: A Design Guide. Butterwoth-Heinemann, 2000.

[2] L. Gibson and M. Ashby, Cellular Solids: Structure and properties. Cambridge University Press, 1997.

[3] T. Lu, H. Stone, and M. Ashby, "Heat transfer in open-cell metal foams," Acta Mater., vol. 46, pp. 3619 - 3635, 1998.

[4] R. Mines, S. Tsopanos, Y. Shen, R. Hasan, and S. McKown, "Drop weight impact behaviour of sandwich panels with metallic micro lattice cores," Int. J. Impact Eng., vol. 60, pp. 120 - 132, 2013. 
[5] A. J. Fuller, T. Kim, H. P. Hodson, and T. J. Lu, "Measurement and interpretation of the heat transfer coefficients of metal foams," P. I. Mech. Eng. C -J. Mec., vol. 219, pp. $183-191,2005$.

[6] F. Han, G. Seiffert, Y. Zhao, and B. Gibbs, "Acoustic absorption behaviour of an open-celled aluminium foam," J. Phys. D: Appl. Phys, vol. 36, pp. 294-302, 2003.

[7] S. G. Mosanenzadeh, H. E. Naguib, C. B. Park, and N. Atalla, "Design and development of novel bio-based functionally graded foams for enhanced acoustic capabilities," J. Mater. Sci., vol. 50, pp. 1248-1256, 2015.

[8] J. Banhart, "Manufacture, characterisation and application of cellular metals and metal foams," Prog. Mater. Sci, vol. 46, pp. 559 - 632, 2001.

[9] H. Wadley, N. Fleck, and A. Evans, "Fabrication and structural performance of periodic cellular metal sandwich structures," Compos. Sci. Technol., vol. 63, pp. 2331 - 2343, 2003.

[10] H. Wadley, "Multifunctional periodic cellular metals," Phil. Trans. R. Soc. $A$, vol. 364 , pp. $31-68,2006$.

[11] J. Wang, A. Evans, K. Dharmasena, and H. Wadley, "On the performance of truss panels with Kagom cores," Int. J. Solids Struct., vol. 40, pp. 6981 $-6988,2003$.

[12] H. Wadley, "Cellular Metals Manufacturing," Adv. Eng. Mater., vol. 4, pp. 726-733, 2002.

[13] B. Wang and G. Cheng, "Design of cellular structures for optimum efficiency of heat dissipation," Struct. Multidiscip. O., vol. 30, pp. $447-458$, 2005.

[14] C. Seepersad, J. Allen, D. McDowell, and F. Mistree, "Multifunctional Topology Design of Cellular Material Structures," ASME. J. Mech. Des., vol. 130, p. 31404, 2008.

[15] A. Evans, J. Hutchinson, N. Fleck, M. Ashby, and H. Wadley, "The topological design of multifunctional cellular metals," Prog. Mater. Sci., vol. 46, pp. $309-327,2001$.

[16] D. Brackett, I. Ashcroft, R. Wildman, and R. Hague, "An error diffusion based method to generate functionally graded cellular structures," Computers 83 Structures, vol. 138, pp. 102 - 111, 2014.

[17] P. Zhang, J. Toman, Y. Yu, E. Biyikli, M. Kirca, M. Chmielus, and A. To, "Efficient Design-Optimization of Variable-Density Hexagonal Cellular Structure by Additive Manufacturing: Theory and Validation," $J$. Manuf. Sci. Eng., vol. 137, pp. 21004 - 21012, 2015. 
[18] D. Brackett, I. Ashcroft, and R. Hague, "Topology Optimization for Additive Manufacturing," in Solid Freeform Fabrication Symposium Proceedings, 2011.

[19] I. Maskery, A. Hussey, A. Panesar, A. Aremu, C. Tuck, I. Ashcroft, and R. Hague, "An investigation into reinforced and functionally graded lattice structures," J. Cell. Plast, pp. 1-15, 2016.

[20] W. van Grunsven, E. Hernandez-Nava, G. C. Reilly, and R. Goodall, "Fabrication and Mechanical Characterisation of Titanium Lattices with Graded Porosity," Metals, vol. 4, pp. 401 - 409, 2014.

[21] Y. Hangai, K. Takahashi, T. Utsunomiya, S. Kitahara, O. Kuwazuru, and N. Yoshikawa, "Fabrication of functionally graded aluminum foam using aluminum alloy die castings by friction stir processing," Mat. Sci. Eng. A - Struct., vol. 534, pp. 716 - 719, 2012.

[22] A. H. Brothers and D. C. Dunand, "Mechanical properties of a densitygraded replicated aluminum foam," Mat. Sci. Eng. A - Struct., vol. 489, pp. $439-443,2008$.

[23] A. Hassani, A. Habibolahzadeh, and H. Bafti, "Production of graded aluminum foams via powder space holder technique," Mater. Design, vol. 40, pp. $510-515,2012$.

[24] H.-P. Heim and M. Tromm, "Injection molded components with functionally graded foam structures Procedure and essential results," J. Cell. Plast., vol. 0, pp. 1 - 21, 2015.

[25] C. Tissandier, R. González-Núñez, and D. Rodrigue, "Asymmetric microcellular composites: Morphological properties," J. Cell. Plast., vol. 50, pp. $449-473,2014$.

[26] X.-c. Zhang, L.-q. An, and H.-m. Ding, "Dynamic crushing behavior and energy absorption of honeycombs with density gradient," J. Sandw. Struct. Mater., vol. 16, pp. 125-147, 2014.

[27] D. Mousanezhad, R. Ghosh, A. Ajdari, A. Hamouda, H. Nayeb-Hashemi, and A. Vaziri, "Impact resistance and energy absorption of regular and functionally graded hexagonal honeycombs with cell wall material strain hardening," Int. J. Mech. Sci., vol. 89, pp. 413-422, 2014.

[28] I. Maskery, A. Aremu, M. Simonelli, C. Tuck, R. Wildman, I. Ashcroft, and R. Hague, "Mechanical Properties of Ti-6Al-4V Selectively Laser Melted Parts with Body-Centred-Cubic Lattices of Varying Cell Size," Exp. Mech., vol. 55, pp. $1261-1272,2015$.

[29] C. Yan, L. Hao, A. Hussein, S. L. Bubb, P. Young, and D. Raymont, "Evaluation of light-weight AlSi10Mg periodic cellular latticestructures fabricated via direct metal laser sintering," J. Mater. Process Tech., vol. 214, pp. 856864, 2014. 
[30] C. Yan, L. Hao, A. Hussein, P. Young, J. Huang, and W. Zhu, "Microstructure and mechanical properties of aluminium alloy cellular lattice structures manufactured by direct metal laser sintering," Mat. Sci. Eng. A - Struct., vol. 628 , pp. $238-246,2015$.

[31] C. Yan, L. Hao, A. Hussein, P. Young, and D. Raymont, "Advanced lightweight 316L stainless steel cellular lattice structures fabricated via selective laser melting," Mater. Design, vol. 55, pp. 533-541, 2014.

[32] J. Brennan-Craddock, D. Brackett, R. Wildman, and R. Hague, "The design of impact absorbing structures for additive manufacture," J. Phys.: Conference Series, vol. 382, p. 012042, 2012.

[33] R. E. Winter, M. Cotton, E. J. Harris, J. R. Maw, D. J. Chapman, D. E. Eakins, and G. McShane, "Plate-impact loading of cellular structures formed by selective laser melting," Model. Simul. Mater. Sc., vol. 22, p. 025021, 2014.

[34] J. Miltz and O. Ramon, "Energy Absorption Characteristics of Polymeric Foams Used as Cushioning Materials," Polym. Eng. Sci., vol. 30, pp. 129 - 133, 1990.

[35] Q. M. Li, I. Magkiriadis, and J. J. Harrigan, "Compressive Strain at the Onset of Densification of Cellular Solids," J. Cell. Plast., vol. 42, pp. 371 $-392,2006$.

[36] N. Aboulkhair, N. Everitt, I. Ashcroft, and C. Tuck, "Reducing porosity in AlSi10Mg parts processed by selective laser melting," Additive Manufacturing, pp. $77-86,2014$.

[37] I. Maskery, N. Aboulkhair, M. Corfield, C. Tuck, A. Clare, R. Leach, R. Wildman, I. Ashcroft, and R. Hague, "Quantification and characterisation of porosity in selectively laser melted AlSi10Mg using X-ray computed tomography," Mater. Charact., vol. 111, pp. 193 - 204, 2016.

[38] N. Aboulkhair, C. Tuck, I. Ashcroft, I. Maskery, and N. Everitt, "On the Precipitation Hardening of Selective Laser Melted AlSi10Mg," Metall. Mater. Trans. A, vol. 46, p. 3337, 2015.

[39] "ASTM E8 / E8M-15a, Standard Test Methods for Tension Testing of Metallic Materials."

[40] N. T. Aboulkhair, I. Maskery, C. Tuck, I. Ashcroft, and N. M. Everitt, "The microstructure and mechanical properties of selectively laser melted AlSi10Mg: The effect of a conventional T6-like heat treatment," Mat. Sci. Eng. A - Struct., vol. 667, pp. 139 - 146, 2016.

[41] M. F. Ashby and R. F. M. Medalist, "The mechanical properties of cellular solids," Metall. Trans. A, vol. 14, pp. 1755-1769, 1983. 
[42] R. Hasan, R. Mines, E. Shen, S. Tsopanos, and W. Cantwell, "Comparison on Compressive Behaviour of Aluminium Honeycomb and Titanium Alloy Micro Lattice Blocks," Key Eng. Mat., vol. 462-463, pp. 213-218, 2011.

[43] C. Qiu, S. Yue, N. J. Adkins, M. Ward, H. Hassanin, P. D. Lee, P. J. Withers, and M. M. Attallah, "Influence of processing conditions on strut structure and compressive properties of cellular lattice structures fabricated by selective laser melting," Mat. Sci. Eng. A - Struct., vol. 628, pp. 188 197, 2015.

[44] C. Yan, L. Hao, A. Hussein, and P. Young, "Ti6Al4V triplyperiodic minimalsurface structuresfor bone implants fabricated via selective laser melting," J. Mech. Behav. Biomed. Mater., vol. 51, pp. 61-73, 2015.

[45] S. V. Bael, G. Kerckhofs, M. Moesen, G. Pyka, J. Schrooten, and J. Kruth, "Micro-CT-based improvement of geometrical and mechanical controllability of selective laser melted Ti6Al4V porous structures," Mat. Sci. Eng. A - Struct., vol. 528, pp. 7423 - 7431, 2011.

[46] S. McKown, Y. Shen, W. Brookes, C. Sutcliffe, W. Cantwell, G. Langdon, G. Nurick, and M. Theobald, "The quasi-static and blast loading response of lattice structures," Int. J. Impact Eng., vol. 35, pp. 795-810, 2008.

[47] J. Li, G. Ma, H. Zhou, and X. Du, "Energy Absorption Analysis of Density Graded Aluminium Foam," Int. J. Protect Struct., vol. 2, pp. 333-349, 2011.

[48] Z. Wang, Z. Li, J. Ning, and L. Zhao, "Effect of heat treatments on the crushing behaviour and energy absorbing performance of aluminium alloy foams," Mater. Design, vol. 30, pp. 977 - 982, 2009.

[49] O. Olurin, N. Fleck, and M. Ashby, "Deformation and fracture of aluminium foams," Mat. Sci. Eng. A - Struct., vol. 291, pp. 136-146, 2000.

[50] S. Maiti, L. Gibson, and M. Ashby, "Deformation and energy absorption diagrams for cellular solids," Acta Met., vol. 32, pp. 1963 - 1975, 1984. 